\title{
Energy Partition and Segregation for an Intruder in a Vibrated Granular System under Gravity
}

\author{
J. Javier Brey, ${ }^{*}$ M. J. Ruiz-Montero, and F. Moreno \\ Física Teórica, Universidad de Sevilla, Apdo. de Correos 1065, E-41080 Sevilla, Spain
}

(Received 22 April 2005; published 23 August 2005)

\begin{abstract}
The difference of temperatures between an impurity and the surrounding gas in an open vibrated granular system is studied. It is shown that, in spite of the high inhomogeneity of the state, the temperature ratio remains constant in the bulk of the system. The lack of energy equipartition is associated to the change of sign of the pressure diffusion coefficient for the impurity at certain values of the parameters of the system, leading to a segregation criterium. The theoretical predictions are consistent with previous experimental results, and also in agreement with molecular dynamics simulation results reported in this Letter.
\end{abstract}

DOI: 10.1103/PhysRevLett.95.098001

Granular systems, i.e., assemblies of macroscopic grains dissipating part of their kinetic energy during collisions, exhibit a rich phenomenology with many qualitative differences as compared with molecular systems [1]. Among these are the breakdown of energy equipartition $[2,3]$ and particle segregation in agitated mixtures [4,5]. The aim of this work is to investigate both effects for a dilute mixture in the tracer limit, i.e., very low mole fraction of one of the species, showing that there is a close relationship between them.

Different species of a granular mixture have different granular temperatures, defined from the average kinetic energy of the particles. A prediction for their ratio in a dilute binary mixture of smooth inelastic hard spheres has been derived from the inelastic Boltzmann equations describing the time evolution of the distribution functions of the species [6], and its accuracy has been verified by molecular dynamics simulations for the particular case of an isolated homogeneous system [7]. In this Letter, it will be shown that kinetic theory also accurately predicts the temperature ratio in the case of a highly inhomogeneous driven system. This ratio turns out to be constant in the bulk of the system, consistent with the experimental results reported in [3]. As in the homogeneous state, deviations from equipartition depend on the mechanical differences between the species and the degrees of inelasticity of collisions.

Particle segregation is the demix of a granular mixture when shaken. Usually, the larger particles are observed to rise (Brazil-nut effect), although under certain conditions they can also tend to descend (reverse Brazil-nut effect). Several mechanisms, corresponding to different scenarios, have been proposed to explain both behaviors $[4,8,9]$, although the phenomenon is far from being fully understood. Here, segregation will be investigated in the context of hydrodynamics for a dilute granular mixture as derived from kinetic theory [10]. The relative position of the tracer component with respect to the excess component is determined by the sign of the pressure diffusion coefficient. While in a molecular gas this sign is fixed by the mass ratio of the particles of the components, for a granular gas
PACS numbers: 45.70.Mg, 05.20.Dd

it also depends on the temperature ratio. Because of the lack of energy equipartition, if follows that the criterion for segregation is rather complicated, involving all the parameters of the mixture.

The model system considered is a low density gas of smooth inelastic hard spheres $(d=3)$ or disks $(d=2)$ of mass $m$ and diameter $\sigma$, and one impurity of mass $m_{0}$ and diameter $\sigma_{0}$. This is formally equivalent to the tracer limit for the impurity component. Inelasticity of collisions is specified by two independent constant coefficients of normal restitution, $\alpha$ and $\alpha_{0}$, referring to gas-gas and impurity-gas collisions, respectively. The system is in presence of a uniform external field of the gravitational type, so each particle is submitted to a force per unit of mass given by $-g_{0} \hat{\boldsymbol{e}}_{z}$, where $g_{0}$ is a positive constant and $\hat{\boldsymbol{e}}_{z}$ a unit vector in the direction of the $z$ axis. Energy is continuously supplied to the system through the bottom wall located at $z=0$ that is vibrating with small amplitude and high frequency. There is no upper wall, i.e., the system is open.

Under the above conditions, the system exhibits an inhomogeneous steady state with gradients only in the $z$ direction and vanishing velocity field. In the case of a onecomponent system, it has been verified that the hydrodynamic profiles away from the walls are well described by the hydrodynamic equations [11,12]. It is assumed that these profiles are not affected by the inclusion of the impurity. Consider the local temperatures of the gas and the impurity, $T(z)$ and $T_{0}(z)$, defined in the usual way from the respective mean square velocities (with the Boltzmann constant set equal to unity). A formal relation between both temperatures can be derived if the existence of a hydrodynamic regime is assumed and, moreover, that the corresponding "normal" solution of the Boltzmann equation for the impurity can be generated by an extension of the Chapman-Enskog method $[10,13]$. Then, it is obtained that the lowest order in the gradients of the cooling rates for the gas, $\zeta^{(0)}(z)$, and the impurity, $\zeta_{0}^{(0)}(z)$, must be equal. These rates are nonlinear functionals of the zeroth order distribution functions, and can be estimated at good ap- 
proximation using Maxwellians, with the results [14]

$$
\begin{gathered}
\zeta^{(0) *} \equiv \frac{\zeta^{(0)}(z)}{n(z) \sigma^{d-1} v_{g}(z)}=\frac{\sqrt{2} \pi^{(d-1) / 2}}{\Gamma(d / 2) d}\left(1-\alpha^{2}\right), \\
\zeta_{0}^{(0) *} \equiv \frac{\zeta_{0}^{(0)}(z)}{n(z) \sigma^{d-1} v_{g}(z)}=\nu_{0}^{*}(1+\phi)^{1 / 2}\left(1-h \frac{1+\phi}{\phi}\right),
\end{gathered}
$$

where $n(z)$ is the number density of the gas, $v_{g}(z)=$ $[2 T(z) / m]^{1 / 2}, h=m\left(1+\alpha_{0}\right) / 2\left(m+m_{0}\right), \nu_{0}^{*}$ is a dimensionless impurity-particle collision rate,

$$
\nu_{0}^{*}=\frac{8 h \pi^{(d-1) / 2}}{\Gamma(d / 2) d}\left(\frac{\bar{\sigma}}{\sigma}\right)^{d-1},
$$

with $\bar{\sigma}=\left(\sigma+\sigma_{0}\right) / 2$, and $\phi$ the ratio of mean square velocities,

$$
\phi=\frac{m T_{0}(z)}{m_{0} T(z)} .
$$

Equating Eqs. (1) and (2) provides the equation for $\phi$,

$$
\begin{gathered}
(1+\phi)^{1 / 2}\left(1-h \frac{1+\phi}{\phi}\right)=\frac{\beta}{h}, \\
\beta \equiv \frac{1-\alpha^{2}}{4 \sqrt{2}}\left(\frac{\sigma}{\bar{\sigma}}\right)^{d-1} .
\end{gathered}
$$

This gives a cubic equation which has a unique real, positive solution for all the allowed values of $h$ and $\beta$. For elastic collisions one gets $\phi=m / m_{0}$, as required by energy equipartition. Moreover, in the limit of an elastic gas, $\alpha=1$, but inelastic collisions between the intruder and the fluid, $\alpha_{0}<1$, the expression obtained in Ref. [15] is recovered. The behavior of the solution in the limit $\phi \rightarrow$ 0 in the context of the isolated homogeneous state has been analyzed in [16], where it has been shown that a qualitative change similar to a second order phase transition occurs, but this will not be discussed here.

From Eq. (5) it follows that $\phi$ does not depend on $z$. Therefore, the temperature ratio $T_{0}(z) / T(z)$ not only differs in general from unity, but remains constant along the system. Moreover, it does not depend either on the precise way in which the wall is being vibrated, as long as the assumed steady state is reached. Of course, the result only holds in the bulk region of the system, where a hydrodynamic description is expected to apply. These predictions are in qualitative agreement with the experimental results found by Feitosa and Menon [3], although it must be realized that their experiments are not carried out in the tracer limit. On the other hand, these authors did not find any dependence of the temperature ratio on the inelasticity of the grains, while Eq. (5) predicts a rather strong one.

To check the above results, we have performed molecular dynamics (MD) simulations of a system of $N$ inelastic hard disks $(d=2)$. The wall at the bottom vibrates with a sawtooth profile with velocity $\boldsymbol{v}_{w}$ and collisions of the particles with it are elastic. Moreover, the amplitude of the wall motion is much smaller than the mean free path of the particles next to it, so in practice the position of the wall can be taken as fixed at $z=0$. Periodic boundary conditions are used in the direction perpendicular to the field. The units are defined by $m=\sigma=g_{0}=1$. In all the results to be reported in the following, the parameters determining the state of the gas have been kept constant, namely $N=359, \alpha=0.95, v_{w}=5$, and a width $S=50$. For these values, it is verified that the system is fluidized and actually reaches the steady state assumed above. In Fig. 1, the temperature profiles of the impurity for $m_{0} / m=$ $1 / 2, \sigma_{0}=1$, and several values of $\alpha_{0}$ are shown. Also, the temperature profile of the gas (solid line) is included. It is clearly observed that energy equipartition is not verified, and that the temperature difference depends on $\alpha_{0}$. The ratio between the impurity and gas temperatures for the same states is plotted in Fig. 2. In agreement with the theoretical prediction, the ratio remains constant, aside from statistical fluctuations, in the interior of the system, even in the region where each of the partial temperatures presents large gradients. In fact, the kinetic boundary layer next to the vibrating wall is quite narrow. Similar behaviors have been found for other values of $m_{0}$, namely $m_{0} / m=$ $0.75,1$, and 2 .

The quantitative comparison of the simulation results with Eq. (5) is presented in Fig. 3, where $\phi$ is plotted against the parameter $h$. The solid line is the solution of the equation and the different symbols correspond to different values of the mass ratio as indicated. A very good agreement is obtained. It is worth to stress that the theory remains accurate up to very small values of the coefficient

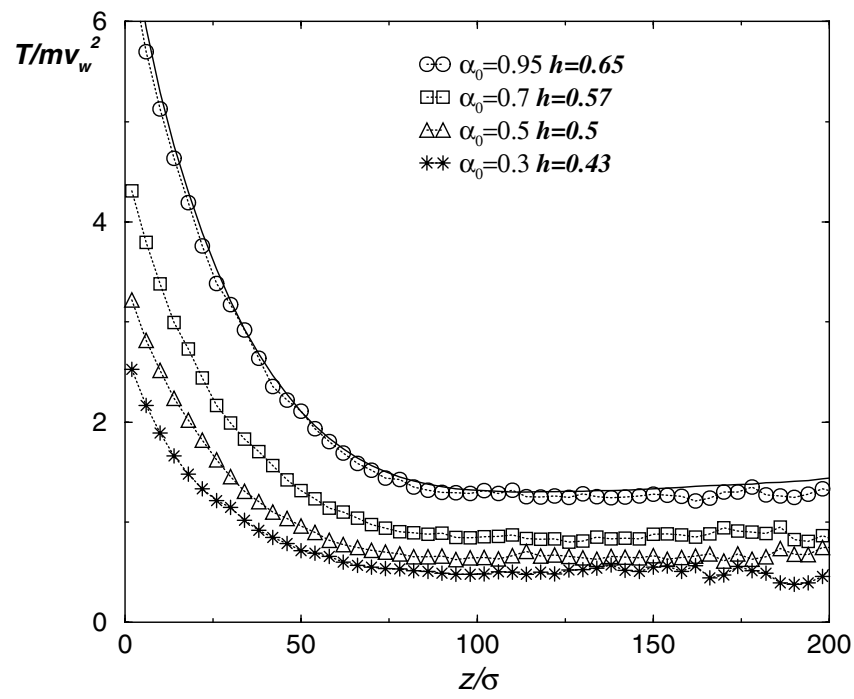

FIG. 1. Dimensionless temperature profiles of the gas (solid line) and the impurity (symbols) for $m_{0} / m=1 / 2$. The different symbols correspond to different values of the restitution coefficient $\alpha_{0}$, as indicated. The values of the parameter $h$, defined in the main text, are also indicated for reference. 


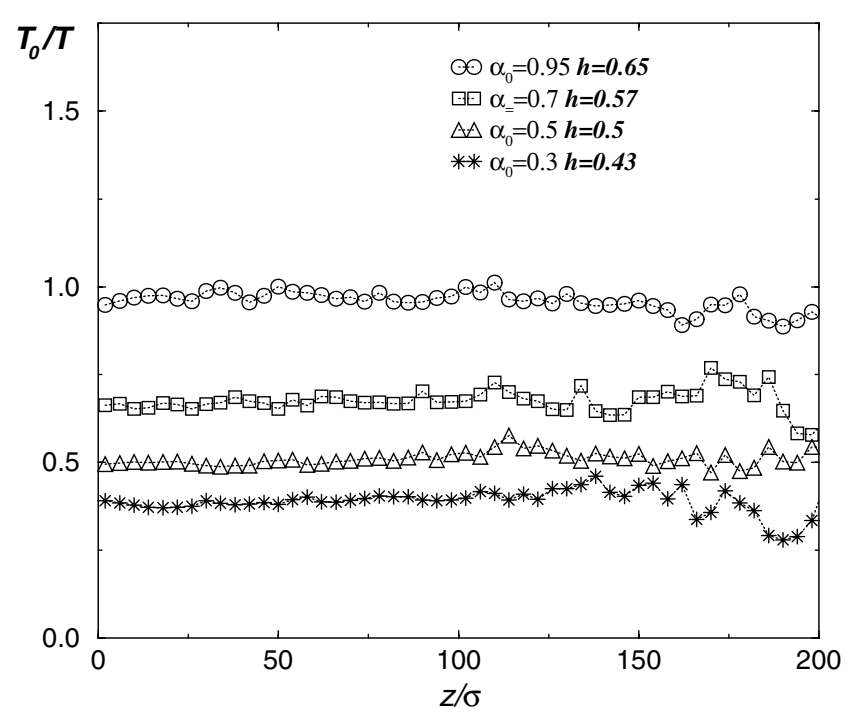

FIG. 2. Profiles of the ratio between the impurity and the gas temperature for the same values of the parameters as in Fig. 1.

of restitution $\alpha_{0}$, indicating the validity of the kinetic and hydrodynamic descriptions even for very strong dissipation. Also, for later discussion note that $\phi$ can be larger than unity even if $m / m_{0} \leq 1$, and vice versa.

Consider next the number density for the impurity in the steady state, $n_{0}(z)$. It is determined by the condition that the associated flux $j_{z}$ must vanish. To first order in the gradients, it is

$$
j_{z}=-m_{0} D \partial_{z} x_{0}-\frac{m n}{T(z)} D^{\prime} \partial_{z} T-\frac{m}{T(z)} D_{p} \partial_{z} p(z),
$$

where $x_{0}=n_{0} / n, p=n T$ is the pressure, $D$ the diffusion coefficient, $D^{\prime}$ the thermal diffusion coefficient, and $D_{p}$ the pressure diffusion coefficient. Explicit expressions for these transport coefficients can be derived by the Chapman-Enskog procedure in the first Sonine approximation $[10,13]$,

$$
\begin{gathered}
D=\frac{n T_{0}}{m_{0}}\left(\nu-\frac{\zeta^{(0)}}{2}\right)^{-1}, \quad D^{\prime}=-\frac{\zeta^{(0)}}{2 \nu} D_{p}, \\
D_{p}=\frac{n_{0} T_{0}}{m n} \frac{\phi-1}{\phi}\left(\nu-\frac{3 \zeta^{(0)}}{2}+\frac{\zeta^{(0) 2}}{2 \nu}\right)^{-1} .
\end{gathered}
$$

Here $\nu$ is a collision frequency,

$$
\nu=\nu_{e} \frac{1+\alpha}{2}(1-\Delta)^{1 / 2}(1+\phi)^{1 / 2},
$$

where $\Delta=m /\left(m+m_{0}\right)$ and $\nu_{e}$ is the elastic limit,

$$
\nu_{e}=\frac{4 \sqrt{2} \pi^{(d-1) / 2}}{\Gamma(d / 2) d} \bar{\sigma}^{d-1} \Delta^{1 / 2} n\left(\frac{T}{m_{0}}\right)^{1 / 2} .
$$

Although the equation $j_{z}=0$ can be (numerically) integrated to get $n_{0}(z)$, given that the hydrodynamic profiles for the gas are known, we will restrict ourselves to a qualitative property of $n_{0}(z)$. We will determine the posi-

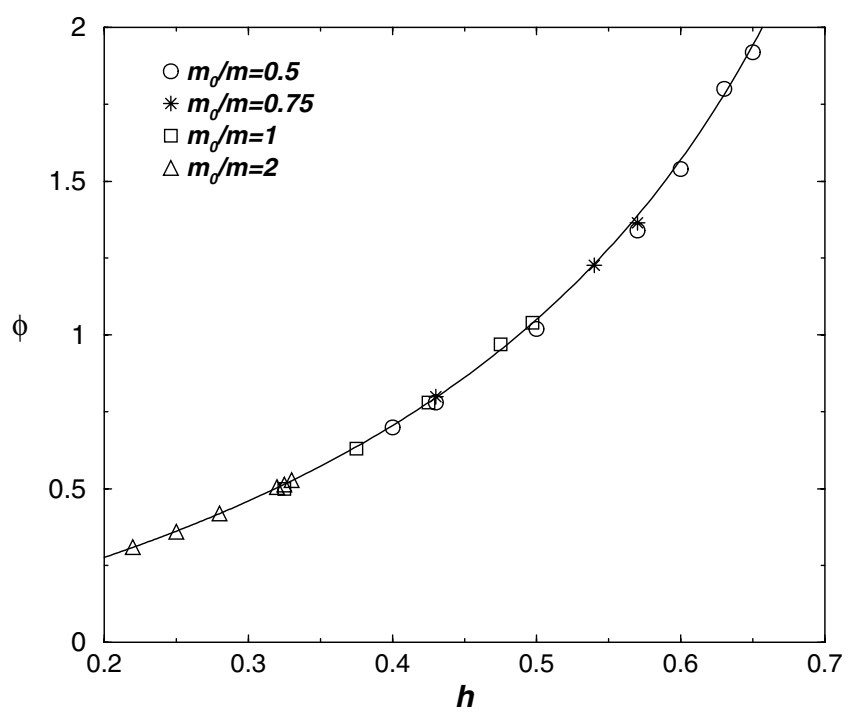

FIG. 3. Ratio of the mean square velocities $\phi$ as a function of the parameter $h$ defined in the main text. The solid line is the theoretical prediction given by Eq. (5) and the symbols are from the simulations for different values of the mass ratio, as indicated.

tion of the maximum of $n_{0}(z)$ relative to that of $n(z)$. That means that we consider situations in which the latter exists, which is always the case for large enough number of particles $N$ [11]. Let us denote by $z_{m}$ the height at which this maximum occurs. It has been established that at this point the temperature of the gas is a decreasing function of $z$, i.e., $\partial T / \partial z<0$ at $z=z_{m}$ [11]. Then, it is obtained

$$
\begin{aligned}
\left(\frac{\partial \ln n_{0}}{\partial z}\right)_{z_{m}}= & \frac{\phi-1}{\phi}\left(1-\frac{\zeta^{(0)}}{2 \nu}\right)^{2}\left(1-\frac{3 \zeta^{(0)}}{2 \nu}+\frac{\zeta^{(0) 2}}{2 \nu^{2}}\right)^{-1} \\
& \times\left|\frac{\partial \ln T}{\partial z}\right|_{z_{m}} .
\end{aligned}
$$

If the right-hand side of the above equation is positive, $n_{0}(z)$ is still growing at $z=z_{m}$, following that its maximum occurs at $z_{0, m}>z_{m}$. In the opposite case, the maximum of $n_{0}(z)$ takes place at $z_{0, m}<z_{m}$. Note that this sign is the same as that of the pressure diffusion coefficient $D_{p}$. The general discussion is rather complicated, given the large number of parameters involved [13]. For the sake of simplicity, we are going to consider the region of the parameter space verifying $\beta<h / 2$, which includes all the MD simulations reported here. For this range of values, it is easily verified that $1-3 \zeta^{(0)} / 2 \nu+\zeta^{(0) 2} / 2 \nu^{2}>0$, and the sign of $\partial \ln n_{0} / \partial z$ at $z=z_{m}$ is determined by the value of $\phi$. For $\phi>1(\phi<1)$ the derivative is positive (negative) and the position of the density maximum for the impurity is higher (lower) than that for the gas. As mentioned above, the value of $\phi$ can be quite different from the mass ratio due to the different temperatures of the impurity and the gas. Since the value of $\phi$ is a function of all the parameters 


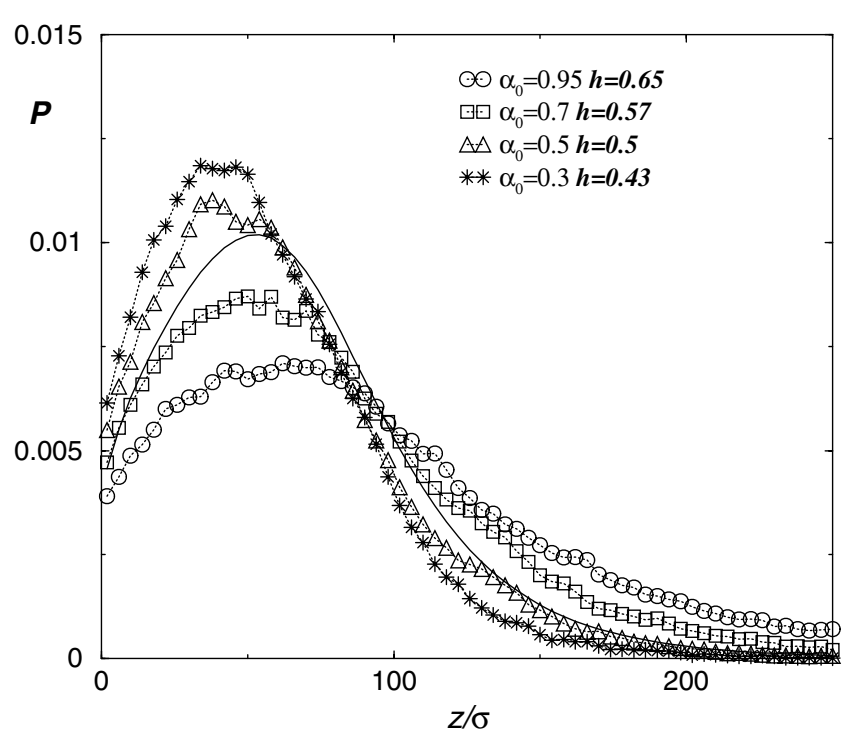

FIG. 4. Normalized height distributions for the gas (solid line) and the impurity (symbols) for the same systems as in Fig. 1.

of the system, the segregation criterion, understood as a rule determining the relative position of the impurity with respect to the gas, also involves all those parameters.

The MD density profiles for the same systems as in Fig. 1 are given in Fig. 4. Again, the solid line is the density profile of the gas, normalized to unity for comparison purposes. As the restitution coefficient $\alpha_{0}$ increases, the maximum of the density profile for the impurity moves towards higher regions and the profile becomes wider, showing the tendency of the impurity to rise. The same effect is observed for the other values of the mass ratio considered. Since the value of $\phi$ increases as $\alpha_{0}$ increases keeping the remaining parameters constant, this behavior is in qualitative agreement with the above theoretical predictions.

The distributions for the position of the impurity become quite flat as the value of $\alpha_{0}$ increases, rendering quite difficult to identify their maximum from the MD results. Then, what has been measured is the center of mass position for both the gas, $z_{\text {c.m. }}$, and the impurity, $z_{0}$. In Fig. 5 the ratio $z_{0} / z_{\text {c.m. }}$ versus $\phi$ is shown. It is seen that the segregation criterium derived above is fairly obeyed, in spite of the fact that the shape of the density distribution clearly indicates that the position of the maxima are rather different from those of the centers of mass. Similar results have been found for other values of the parameters, including different sizes of the impurity [13].

To conclude, it must be stressed that the present work deals with a dilute granular mixture in the tracer limit. At higher densities, other segregation mechanisms, such as those discussed in $[8,9]$, become important. On the other hand, there is no reason to expect that the one identified here fails to be relevant at those densities.

This research was supported by the Ministerio de Educación y Ciencia (Spain) through Grant No. FIS2005-

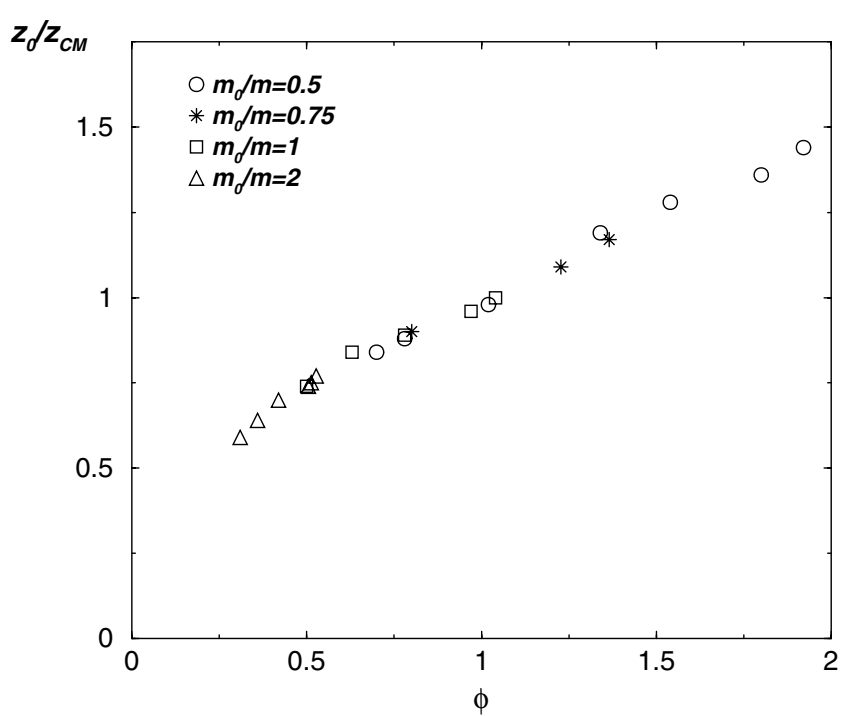

FIG. 5. Ratio between the impurity and the gas center of mass positions vs the ratio of the mean square velocities $\phi$. The different symbols correspond to different values of the mass ratio as indicated.

01398 (partially financed by FEDER funds).

*Electronic address: brey@us.es

[1] See, for instance, H. M. Jaeger, S. R. Nagel, and R. P. Behringer, Rev. Mod. Phys. 68, 1259 (1996).

[2] R. D. Wildman and D. J. Parker, Phys. Rev. Lett. 88, 064301 (2002).

[3] K. Feitosa and N. Menon, Phys. Rev. Lett. 88, 198301 (2002).

[4] J. Duran, J. Rajchenbach, and E. Clément, Phys. Rev. Lett. 70, 2431 (1993).

[5] A. P. J. Breu, H.-M. Ensner, C. A. Kruelle, and I. Rehberg, Phys. Rev. Lett. 90, 014302 (2003).

[6] V. Garzó and J. Dufty, Phys. Rev. E 60, 5706 (1999).

[7] S. R. Dahl, C. M. Hrenya, V. Garzó, and J. W. Dufty, Phys. Rev. E 66, 041301 (2002).

[8] D. C. Hong, P. V. Quinn, and S. Luding, Phys. Rev. Lett. 86, 3423 (2001).

[9] J. T. Jenkins and D. K. Yoon, Phys. Rev. Lett. 88, 194301 (2002).

[10] V. Garzó and J. W. Dufty, Phys. Fluids 14, 1476 (2002).

[11] J. J. Brey, M. J. Ruiz-Montero, and F. Moreno, Phys. Rev. E 63, 061305 (2001).

[12] J. J. Brey and M. J. Ruiz-Montero, Europhys. Lett. 66, 805 (2004).

[13] J. J. Brey, M. J. Ruiz-Montero, and F. Moreno (to be published).

[14] J. W. Dufty, J. J. Brey, and J. Lutsko, Phys. Rev. E 65, 051303 (2002).

[15] P. A. Martin and J. Piasecki, Europhys. Lett. 46, 613 (1999).

[16] A. Santos and J. W. Dufty, Phys. Rev. Lett. 86, 4823 (2001). 